\title{
Technokratie und Freiheit: Zur Ideengeschichte der digitalen Steuerungsutopie
}

\author{
Daniel Schulz
}

Angenommen: 16. November 2021 / Online publiziert: 7. Dezember 2021

(C) Der/die Autor(en) 2021

Zusammenfassung Gegen das präsentistische (Selbst-)Verständnis der Digitalisierung vertritt der Beitrag die These, dass die Entwicklung der digitalen Konstellation eng mit der utopischen Tradition des politischen Denkens verbunden ist. Die Idee einer technischen Steuerung politischer und sozialer Ordnungsfragen ist Ausdruck eines spezifisch modernen Verfügbarkeitsdenkens, das in der Geschichte der politischen Utopien seit Thomas Morus in verschiedenen Varianten durchgespielt wurde. Der Beitrag skizziert in einem ersten Schritt diese Vorgeschichte von der Frühen Neuzeit bis in das zwanzigste Jahrhundert, um dann im zweiten Schritt exemplarisch die behavioristische Utopie B. F. Skinners in diese Entwicklung der technischen Verhaltenskonditionierung einzureihen. Im letzten Teil wird dann gezeigt, wie das digitale Ordnungsdenken der Gegenwart im Zeichen von Big Data erneut an die umfassenden Steuerungshoffnungen der hochmodernen Humantechnik anknüpft. Abschließend wird die Frage gestellt, wie sich die politische Theorie im Deutungskonflikt der Digitalisierung verorten sollte.

Schlüsselwörter Politische Theorie · Digitalisierung · Behaviorismus · Nudging · Technik 


\title{
Technocracy and freedom. The utopias of digital control in the history of political thought
}

\begin{abstract}
Against the presentistic (self-)conception of digitalization, the paper argues that the development of the digital constellation is closely related to the utopian tradition of political thought. The idea of technical steering in political and social matters is the expression of a specifically modern thought of control and has been central to the history of political utopias since Thomas Morus. In a first step, the article sketches this utopian history of technical control from the transition to modernity until the twentieth century. The second step focusses on the example of B. F. Skinner's behaviorist utopia Walden Two, in which key elements of the technocratic tradition come together to form an influential model. The last part shows how the digital age, and the related theoretical discourses connect to this heritage of human techniques. Finally, the paper discusses the question how political theory should position itself within the conflict of interpretating digitalization.
\end{abstract}

Schlüsselwörter Political Theory · Digitalization · Behaviorism · Nudging · Technology

Im Zeitalter der Digitalisierung sind viele alte politische Emanzipationshoffnungen neu geweckt worden: die zahlreichen Innovationen in der gesellschaftlichen Kommunikation, die Umstellung einer one-to-many auf many-to-many Kommunikation, der ungehinderte Zugriff auf Informationen - all diese Aspekte haben die Teilhabeschwellen gesenkt und den Zugang zum öffentlichen Raum erleichtert. Damit erscheint „Vernetzung als die neue Form der Vergemeinschaftung“" (Thiel 2014, S. 468) und bringt die uneingelösten Teilhabeversprechen der Demokratie scheinbar in Reichweite.

Diese transformativen Potenziale der digitalen Technologien wurden vor allem in der Frühzeit des Internets positiv hervorgehoben (Turkle 1984; Weiser 1991; Rheingold 1993; Negroponte 1995, Barlow 1996; Kurzweil 2005). Gleichwohl kann der utopische Aspekt dieser Entwürfe auch aus einer anderen Perspektive beschrieben werden. So lassen sich in der Digitalisierungsdiskussion neben den partizipatorischen Elementen zugleich auch eine ganze Reihe von Vorgriffsversuchen auf die Zukunft aufdecken, die durch die exponentielle Fortschreibung der technischen Steuerungsmöglichkeiten der Gegenwart realisiert werden sollen. Diese auch institutionell durch die TED-Konferenz oder durch das MIT, die Stanford University und andere Formate gerahmten Arbeiten am digitalen Mythos ähneln in dieser Hinsicht auf frappierende Weise dem klassischen Utopiegedanken der europäischen Ideengeschichte - und dieses Verhältnis steht im Mittelpunkt des vorliegenden Beitrages. Die leitende These geht davon aus, dass hinter der Selbstbeschreibung des Digitalisierungsparadigmas als revolutionärer Bruch vielmehr eine Kontinuitätslinie sichtbar gemacht werden kann: Der Anspruch, die Komplexität moderner Gesellschaften durch revolutionäre Technologien steuern zu können, verbindet die Versprechen der digitalen Gegenwart mit den klassischen Utopien der Frühen Neuzeit und der Hochmoderne. Gemeinsam ist diesen Ansätzen zudem, diese Steuerung 
in einem technischen Sinne analog zu Naturbeherrschung zu denken. Dabei wiederholt sich unter dem Stichwort von Big Data das historische Muster, dass mit dem Entstehen neuer Wissensformen - Astronomie, Geometrie, Sozialmathematik, Sozialphysik, Sozialpsychologie, Kognitionswissenschaft - auch die Hoffnung auf eine umfassende gesellschaftliche Gestaltungs- und Steuerungsmöglichkeit genährt wird. Die Digitalisierung erscheint in dieser Perspektive als eine Fortsetzung derjenigen Wissensformen, mit denen gesellschaftliche Ordnungszusammenhänge technisch verfügbar gestellt werden sollen. Die heutigen Digitalisierungsdiskurse sind mit der utopischen Tradition daher über den Technikbegriff verbunden, und zwar in zweifacher Weise: über ihre radikale Fortschritts- und Prognosegläubigkeit auf der einen Seite, und über ihre damit verbundenen technischen Steuerungsansprüche auf der anderen Seite.

Eine der ersten Studien, die diese utopische Dimension des Digitalisierungsdiskurses kritisch analysiert hat, beschreibt die ,californian ideology“ als einen ,,antistatist gospel of cybernetic libertarianism: a bizarre mish-mash of hippie anarchism and economic liberalism beefed up with lots of technological determinism“ (Barbrook und Cameron 1995, S. 6). ${ }^{1}$ Diese frühe herrschaftskritische Analyse setzt sich jedoch nicht mit der Vorgeschichte der Digitalisierung auseinander. Eingelöst wird diese Analyse erst in exemplarischen Fallstudien jüngeren Datums: Jill Lepore hat am konkreten Beispiel der computerbasierten Wahlprognostik und der politischen Kommunikation in den USA gezeigt, wie in der zweiten Hälfte des zwanzigsten Jahrhunderts eine Abwendung vom klassisch-humanistisch geprägten Vernunftideal hin zu einem technischen geprägten Rationalitätskalkül stattfindet - verbunden ist diese Entwicklung unter anderem mit der Kybernetik Norbert Wieners (Lepore 2020; August 2021). Diese „,cold war rationality“ (Erickson et al. 2013) war mit dem Versprechen verbunden, zukünftige Entwicklungen berechenbar und damit einer planenden Steuerbarkeit zugänglich zu machen. Staatstheoretisch ist diese Entwicklung von James C. Scott als technokratische Hochmoderne untersucht worden: Die Verkürzung des aufgeklärten Vernunftbegriffs auf eine instrumentelle Planungs- und Steuerungslogik manifestierte sich neben den von Lepore beschriebenen Bereichen so auch in der Stadtpolitik und der Architektur dieser Zeit (Scott 1999). Während Lepore verdeutlicht, wie die neuen Methoden technischer Verhaltensprognostik und -steuerung zunächst im Bereich der Wahlforschung Anwendung finden, um dann von der amerikanischen Regierung im Vietnam-Krieg im Kampf um Meinungs- und Deutungshoheit eingesetzt zu werden (Lepore 2020, S. $205 \mathrm{ff}$.), so hat Fred Turner bereits 2006 demonstriert, wie sehr auch die amerikanische Gegenkultur von den Entwicklungen der frühen Computertechnologien und ihrem Rationalisierungsideal geprägt worden ist. Das in diesem Kontext verbreitete Idealbild einer dezentralisierten, egalitären, harmonischen und freien Gesellschaft (Turner 2006, S. 1) wurde in zahlreichen alternativen Gemeinschaftsprojekten bereits seit dem Ende der sechziger Jahre an die ermöglichende Potenziale der technischen Entwicklung gebunden. Als Gegenbild zur staatlichen Herrschaftstechnik hat die Alternativkultur somit eine spezifische utopische Vision von Staats- und Politikferne hervorgebracht (ebd., S. 256),

\footnotetext{
1 Zur utopischen Dimension des Marktliberalismus bereits Rosanvallon (1979).
} 
die in den neunziger Jahren die oben genannten „techno-utopians“ des digitalen Zeitalters (ebd., S. 261) nachhaltig beeinflusste.

Wenn also die digitale Revolution der Informations- und Kommunikationstechnologien als Austritt aus der Geschichte und der staatlichen Politik in die „Hypergeschichte“ der „Multiakteurssysteme“ bestimmt wird (Floridi 2015, S. 17 ff. u. S. $221 \mathrm{ff}$.), dann liegen die historischen Spuren zu diesem ahistorischen Selbstbild der digitalen Ordnung in der Utopiegeschichte. Als eine der Ersten ist Shoshana Zuboff diesem Zusammenhang der digitalen Konstellation mit der utopischen Tradition des politischen Denkens nachgegangen. Ihre Analyse des digitalen „,̈berwachungskapitalismus“ (Zuboff 2018) sieht diese neue Ordnung durch die digitale Produktion von Verhaltensdaten geprägt. Sie bilden die Grundlage des Profitmodels von Facebook, Google und Microsoft, die Zuboff als ,angewandte Utopistik“ beschreibt (ebd., S. 470). Die Hauptrolle spielt dabei nicht nur - wie bei Lepore und Turner hervorgehoben - die Kybernetik, sondern auch der Ansatz sozialpsychologischer Konditionierung, den der Behaviorist Burrhus Frederic Skinner in der Mitte des zwanzigsten Jahrhunderts entwickelt hatte und der eine neue Form der weichen Verhaltensmodifikation propagiert (ebd., S. 338).

Der folgende Beitrag will dieser Spur weiter nachgehen und dabei vor allem die politiktheoretische Relevanz dieser Zusammenhänge deutlich machen, die in Zuboffs primär politikökonomisch ausgerichteter Analyse nur angedeutet werden und in den Analysen der hochmodernen Technokratie bislang kaum beachtet wurden. Dazu wird in einem ersten Schritt die Tradition des utopischen Denkens betrachtet. Hier geht es darum, das spannungsreiche Verhältnis zwischen politischem Gestaltungsanspruch und technischer Steuerung herauszustellen, welches die utopische Literaturgattung seit der frühen Neuzeit geprägt hat. Vor diesem Hintergrund wird dann in einem zweiten Schritt deutlich, wie die behavioristische Steuerungsutopie B. F. Skinners an diese utopische Tradition anschließt. Wie dann im letzten Teil deutlich werden soll, setzt das digitale Ordnungsdenken der Gegenwart im Zeichen von Big Data diese umfassenden Kontrollhoffnungen der Hochmoderne fort und stellt erneut die Frage nach dem Verhältnis von technischer Steuerung und politischem Handeln. Dieses Spannungsverhältnis, so die abschließende Überlegung, kann politiktheoretisch als ein Deutungskonflikt verstanden werden, der die gegenwärtigen Gesellschaften im Zeichen der digitalen Transformation prägt.

\section{Zur Utopie der Verhaltenssteuerung}

Digitalisierung lässt sich politiktheoretisch als radikale Verfügbarkeitstechnik interpretieren. Während Digitalisierung im engeren Sinne zunächst nur die Übertragung von Informationen in binär codierte Formate bezeichnet, sind die Konsequenzen dieser Übertragung weitreichend. Nicht nur sinken damit technisch bedingt die Kosten für die Aufzeichnung und Speicherung von Informationen, auch wachsen die Möglichkeiten ihres Abrufs und der Vernetzung - sie werden demnach verfügbar für zahlreiche ökonomische, kulturelle und auch politische Praktiken und Diskurse der Verwertung, der kreativen Neuverknüpfung und der autoritativen Steuerung (Stalder 2016, S. 105). In dieser Hinsicht verändern digitale Techniken die gesell- 
schaftliche Balance von Verfügbarkeit und Unverfügbarkeit, die auch normativ eine beträchtliche Rolle für die Stabilisierung gesellschaftlicher Ordnung spielt. ${ }^{2}$

Technik beschränkt sich seit dem Beginn der Moderne nicht allein auf Naturbeherrschung, sondern dringt auch in den Praxisbereich des Gemeinwesens ein, der in der normativen, aristotelisch von der Vorstellung bürgerschaftlichem Handeln geprägten politischen Philosophie stets gegen eine rein funktionalistische Perspektive abgegrenzt wurde. Damit wird der Begriff der Technik tendenziell unscharf, verliert er doch seine Beschränkung auf den herstellenden Gebrauch von Werkzeugen bzw. die Beherrschung spezifischer Fähigkeiten zur Erzielung eines bestimmten Zweckes und wird zunehmend identisch mit Beherrschung schlechthin. Damit meint Technik nicht mehr nur die technische Herrschaft von Menschen über die Natur, sondern auch die Herrschaft des Menschen über den Menschen. Die Erweiterung technischer Verfügungsgewalt zur „Humantechnik“ (Ellul 1964, S. 319) wird durch die Digitalisierung radikalisiert. Digitale Verhaltensbeobachtung, Verhaltensvorhersage und Verhaltenskontrolle erweitern und entgrenzen den Möglichkeitsraum von Herrschaft - und lassen demokratische Formen des kollektiven Handelns zunehmend als überkommene, ineffiziente Mittel der Entscheidungsfindung erscheinen. Mit diesem „Aberkennen der Bedeutung kollektiver politischer Handlungsformen“ aber knüpft die ,digitale Konstellation“ (Berg et al. 2020, S. 179f.) unmittelbar an die utopische Tradition des politischen Denkens an: Seit seiner Erfindung in der Frühen Neuzeit spielt dieses Genre die Möglichkeit einer perfektionierten Ordnung durch. Die Idee einer umfassenden Verhaltenssteuerung hat hier von Beginn an eine zentrale Rolle eingenommen. Gemeinsames Merkmal der frühneuzeitlichen Utopien ist dabei ein naturwissenschaftliches Verständnis sozialer Ordnung, in der politische Fragen zu technisch lösbaren Problemen transformiert werden. ${ }^{3}$

Politik und Technik stehen jedoch in keinem prinzipiellen Gegensatz. Aus der aristotelischen Perspektive wurde Politik zunächst als spezifisch selbstzweckhafte und autonome Form der Praxis gegen die herstellenden Formen des menschlichen Handelns gestellt (Aristoteles 1969, S. 5 ff.; Arendt 1981). Gegenüber dieser Abgrenzung haben insbesondere moderne Politikbegriffe auch ein instrumentelles, macht- bzw. ressourcendominiertes Verständnis von Politik entwickelt (Machiavelli 1990, Laswell 1936; zu den verschiedenen Facetten des Politikbegriffs Rohe 1994). Dieses spezifisch moderne Politikverständnis betont damit analog zum Begriff der Technik das Moment der Herrschaftssteigerung durch den Einsatz effizienter Mittel und kann somit als eine Angleichung politischen Handelns an technisches Handeln verstanden werden. Auf den ersten Blick erscheint diese technisch gesteigerte Verfügungsmacht als Steigerung gesellschaftlicher Autonomie. Technisierung wirkt aber zugleich in die entgegengesetzte Richtung, wie Hans Blumenberg bereits 1951 gezeigt hat: „Als Grundzug der technischen Sphäre enthüllt sich mehr und mehr ihre Autonomie, die zunehmende Unverfügbarkeit für den Menschen, das Überspielen seiner Entschlüsse, Wünsche, Bedürfnisse durch eine Dynamik der Sache, die dem gesamten Leben der Epoche einen unverkennbaren homogenen Stil aufprägt"

\footnotetext{
2 Vgl. zum Begriff der Unverfügbarkeit nur die Überlegungen bei Vorländer (2013), Dreischer et al. (2013) sowie Rosa (2018).

${ }^{3}$ Zur Utopieliteratur vgl. Saage (1997 und 2002), Voßkamp (1985), Manuel und Manuel (1979).
} 
(Blumenberg 2015, S. 18). Helmuth Schelsky hat diesen Wandel der Technik von Effizienzsteigerung politischer Entscheidung hin zu deren faktischen Einschränkung als Logik des „Sachgesetzlichkeit“ im ,technischen Staat“ analysiert. ${ }^{4}$ Damit aber ist die moderne Technisierung des Politischen Ausdruck eines gesellschaftlichen Autonomiewillens und zugleich Begrenzung seiner Möglichkeit.

Diese Spannung zwischen technischer Ermächtigung und Freiheitsbegrenzung ist bereits in der neuzeitlichen Utopietradition angelegt. Utopien sind seit Thomas Morus nicht nur politische Entwürfe, sondern verdanken einen großen Teil ihrer Ordnungsvorstellungen dem naturwissenschaftlichen Weltbild der Neuzeit. Dessen Begriffe von Gesetzmäßigkeit und Naturbeherrschung sind im utopischen Genre auf die menschliche Ordnung übertragen worden, welche nun auf eine neue, wissenschaftliche und rationale Weise als perfektes Ensemble entworfen werden kann. Nach der Verzeitlichung des Utopiegedankens liegt die perfekte Ordnung nicht mehr auf einer Insel oder an einem unzugänglichen, verborgenen Ort, sondern in der auf der Zeitachse zu erreichenden, zukünftigen Gegenwart (Koselleck 1985). Ebenso wie in den Digitalisierungsdiskursen ragt die Zukunft immer schon in das Heute hinein und kann von einer (Cyber-)Avantgarde vorweggenommen werden.

Die Ursprünge dieser Tradition gehen jedoch zurück auf die antike politische Philosophie: Platons Politeia ist die erste Staatsform, in der ,das menschliche Miteinander technisch geregelt werden kann“ (Arendt 1981, S. 289). Das utopische Genre hat sich daher auch nicht zufällig im Rahmen der frühneuzeitlichen Platonrezeption fortentwickelt. Thomas Morus Utopia (1516) mag wohlmöglich nur als „Humanistenscherz“ gemeint gewesen sein (Ottmann 2006, S. 138) - mit seinen innerweltlichen Wissensbezügen stellte der literarische Entwurf aber eine Alternative zu den apokalyptischen Endzeiterwartungen dar, wie sie im Zeitalter der Reformation allgegenwärtig waren. ${ }^{5}$ Die enge Verbindung von politisch-sozialer Neugestaltung und wissenschaftlichem Ordnungswissen bewegt sich in der frühen Neuzeit zunehmend vom philosophischen Humanismus zu den im Entstehen begriffenen Naturwissenschaften. ${ }^{6}$ Mit der Verschiebung zum neuzeitlichen Wissenschaftsverständnis wird die Welt nicht mehr vermittels philosophischer oder theologischer Abhandlungen lesbar, sondern durch die experimentelle Lektüre im „Buch der Natur“ (Blumenberg 1983, S. 61): An die Stelle der Bibliothek als Wissenslieferant tritt das Labor, und das Experiment löst die philosophische Reflexion als primäre Quelle von Erkenntnis ab.

Die Utopien der Frühen Neuzeit sind somit erste Zeugnisse eines neuzeitlichen, sozialtechnologischen Planbarkeits- und der Optimierungsglaubens. Politisches Handeln im Sinne einer an Freiheit und Autonomie orientierten aristotelischen Praxis

\footnotetext{
${ }^{4}$ Diese Diagnose wird scharf kontrastiert mit dem legitimationsstiftenden Selbstbild der Demokratie: „An die Stelle eines politischen Volkswillens tritt die Sachgesetzlichkeit“ (Schelsky 1979, S. 465). Damit setzt die Technisierung des Sozialen auch die mit den Begriffen Staat und Politik verbundenen kollektiven Steuerungsvorstellungen unter den Druck abnehmender Evidenz - zu dieser Debatte, die vor allem über die Systemtheorie Luhmanns vermittelt wurde, vgl. Nassehi (2002) und Lange (2002).

5 Zur „Glut apokalyptischer Weltdeutungen“ in der ersten Hälfte des sechzehnten Jahrhunderts vgl. Kaufmann (2017, S. 353).

6 Hier wäre näher einzugehen auf Campanellas Sonnenstaat (1602), Andreaes Christianopolis (1619) und Bacons Nova Atlantis (1624). Als Überblick vgl. Ottmann (2006, 138ff.).
} 
wird damit nicht vollkommen verdrängt, tritt aber in ein Spannungsverhältnis zu einem an wissenschaftsbasierter Naturbeherrschung angelehnten Verständnis von Politik. Zumeist steht nicht die politische Staatsverfassung selbst im Mittelpunkt der utopischen Entwürfe, sondern die vielmehr die detaillierte Gestaltung aller - auch und gerade der privaten - Aspekte des gemeinschaftlichen Zusammenlebens: Organisation und Planung des Arbeitslebens, der Familienstruktur, der gemeinsamen Mahlzeiten, der arbeitsfreien Zeit etc., sowie der dazu notwendigen städtebaulichen Strukturen (zu letzterem Kruft 1989).

Politiktheoretisch wird dieser Umbruch in mehrfacher Hinsicht reflektiert bei Niccolò Machiavelli. Trotz seiner engen Verbindung zur humanistisch-republikanischen Tradition hat gerade die Reformulierung von Politik als instrumentell kalkulierende Herrschaftstechnik das neuzeitliche Verständnis nachhaltig geprägt (Skinner 1998, S. $180 \mathrm{ff}$.). In der Folge wird politisches Handeln immer auch zu einer Frage der strategischen Machttechnik - dabei geht es nicht nur um die Frage der direkten Herrschaftsmittel und ihrer Monopolisierung, wie sie im Mittelpunkt der Rekonstruktion von Max Weber stand (Weber 2009). Auch die indirekte Steuerung über Stimuli wird im Sinne der politischen Verhaltenssteuerung erwogen - beispielsweise in Gabriel Naudés Considérations politiques sur les coups d'État (1667), die die Schaffung von positiven und die Vermeidung von negativen Handlungsanreizen zum Gegenstand politischer Klugheit machen. ${ }^{7}$ Der Politikbegriff weist in der Frühen Neuzeit somit eine Bedeutungsverschiebung auf: Sein normativer, bürgerschaftlicher Ordnungshorizont wurde zunehmend von der Vorstellung souveräner staatlicher Herrschaft überlagert. Im Zuge dieser Entwicklung wird Politik dann auch zu einem Instrument der Verhaltensbeeinflussung Dritter.

Damit bewegt sich das neuzeitliche Denken in eine Richtung, in der die Natur und ihre Beherrschung zum zentralen Objekt des wissenschaftlichen Diskurses wird. Insoweit sich aber die Natur diesen Bemühungen einer vollkommenen Beherrschung entzieht und daher dem menschlichen Zugriff niemals in Gänze verfügbar wird, erscheint für Descartes und darüber hinaus ,,das Reich der Konstruktion, die technische Welt“ zunehmend als der wahre „Bereich unserer Souveränität und Freiheit" (Blumenberg 2015, S. 78). Erst in der konstruktiven Überwindung der Natur erfährt sich der Mensch demnach als wahrhafter Schöpfer. Das zu diesem mechanistischen Weltmodell passende politische Ordnungsmodell hat der ehemalige Sekretär von Francis Bacon, Thomas Hobbes, 1651 entworfen: Der more geometrico konstruierte Maschinenstaat schließt insofern nicht nur wissenschaftstheoretisch an die frühneuzeitlichen Steuerungsutopien an. Auch inhaltlich geht es darum, das komplexe Geflecht der Affekte auf Grundlage eines wissenschaftlichen Kalküls zu rationalisieren. Der zu diesem Zweck entworfene Staatsautomat dient dabei nicht allein der Produktion von sicherheitsstiftenden Gesetzen, sondern vornehmlich da$\mathrm{zu}$, politische Stabilisierung durch die symbolisch-visuelle Induktion von Angst vor

\footnotetext{
7 ,un choix et triages des choses qui sont á fuir, ou à désirer“, Naudé (2004, S. 85).
} 
Bestrafung zu erreichen. ${ }^{8}$ Hobbes' Leviathan ist damit insofern Teil der technischen Naturbeherrschung, als es sich hier nicht nur um die Beherrschung der Natur durch den Menschen, sondern vor allem auch um die technische Beherrschung der menschlichen Natur handelt. Mit Thomas Hobbes wird damit der moderne Staat selbst als eine „Humantechnik“ (Ellul 1964, S. 319) begründet. Der Leviathan ist eine affektbasierte, die Leidenschaften kontrollierende und zähmende politische Maschine, die dem Zweck öffentlicher Sicherheitsproduktion dient (Bredekamp 2003; Frankenberg 2010; Bates 2012; Skinner 2017; Schulz 2017). Dieses Verständnis setzte sich schließlich in der Idee der absolutistischen Staatsmaschine durch (Stollberg-Rilinger 1986).

Während also die mechanische Steuerung der Leidenschaften zum großen Projekt absolutistischer Souveränität entwickelt wird, so finden Planung und Optimierung von Politik und Gesellschaft auch Eingang in die politischen Theorien und konkreteren Verfassungsprojekte der Aufklärung. ${ }^{9}$ Technische Mechanisierung der politischen Ordnung und aufklärerisches Perfektionsideal verbinden sich seit dem achtzehnten Jahrhundert zu einer Vorstellung, die in der perfekten Gestaltung des politischen Gemeinwesens eine notwendige Voraussetzung der menschlichen Selbstperfektionierung sah. Im Lichte einer allgemeinen Faszination dieser Zeit mit Automaten ${ }^{10}$ konnte diese Verbindung auch zu einer direkten Anwendung technischmathematischer Modellvorstellung auf die Gestaltung von Staat und Gesellschaft führen (Condorcet 1993, ders. 2010, S. 173 ff. u. S. 171).

Damit aber entwickelt sich die Ambivalenz zwischen Freiheit und Sozialtechnologie mitten im Denken der Aufklärung selbst. Das neunzehnte Jahrhundert hat mit der zunehmenden lebensweltlichen Bedeutung von Wissenschaft und Industrie auch eine ganze Reihe von entsprechenden Utopien hervorgebracht, in denen sich die Anwendung technischer Problemlösungen zu einer reißbrettartigen Gesamtskizze neuer Gesellschaftsmodelle verdichtet. Während in der Folge von Auguste Comtes Positivismus und der im Entstehen begriffenen Wissenschaft von der Gesellschaft bei Adolphe Quetelet die Möglichkeit einer ,,physique sociale“ ausgelotet wird (Quetelet 1835), schreiben zahlreiche Utopisten die mit Morus begonnene Tradition im Kontext der industriellen Moderne fort: Beginnend mit Louis-Sébastien Merciers L'an deux mille quatre cent quarante (1771) setzte die Verzeitlichung der Utopie ein, mit der die geplante Ordnung in die Zukunft verlegt wird. Damit steigt zugleich die Erwartung an die tatsächliche und konkrete Umsetzbarkeit des utopischen Modells. Die Entwürfe von Saint-Simon, Charles Fourier und Robert Owen verlieren

\footnotetext{
8 „Letzte Ursache, Zweck oder Absicht der Menschen (...) bei der Einführung jener Selbstbeschränkung ist die Vorsorge für ihre Selbsterhaltung und dadurch für ein zufriedenes Leben; das heißt, daß sie jenem elenden Kriegszustand entkommen wollen, der (...) die notwendige Folge der natürlichen Gemütsbewegungen der Menschen ist, wenn es keine sichtbare Macht gibt, um sie in Schrecken zu halten und sie durch Furcht vor Strafe an die Erfüllung ihrer Verträge und die Befolgung jener Naturgesetze zu binden (...)“ (Hobbes 1996, S. 141).

9 So beispielsweise auf der Grundlage des „Perfectio-Ideals“ bei Turgot und dem Abbé Saint Pierre (Koselleck 1985, S. 5), bzw. mit dem Begriff der perfectibilité (Rousseau 1995, S. 107 ff.).

${ }^{10}$ Die „,mechanische Ente“ von Jacques de Vaucanson (1738) und der „Schachtürke“ von Wolfgang von Kempelen (1769) sind nur die bekanntesten Beispiele; vgl außerdem L'Homme-Machine von Julien Offroy de La Mettrie (1748).
} 
daher zunehmend die literarische Form einer Erzählung von einem fiktiven Ort und nehmen die Form eines noch in der eigenen Lebenszeit realisierbaren Projektes an. ${ }^{11}$ Damit handelt es sich hier bereits um explizite Steuerungsutopien einer politischen Avantgarde, die über die technologischen Einblicke in die gesellschaftlichen Kausalzusammenhänge verfügt und daraus einen konkreten Handlungs- und Gestaltungsanspruch ableitet. ${ }^{12}$

Die Vorstellung einer wissenschaftlichen Rationalisierung der Politik bildet vor diesem Hintergrund eine der Grundströmungen des neunzehnten Jahrhunderts. Während einerseits im nachrevolutionären Liberalismus die Idee einer Souveränität der Vernunft als vermeintlicher Ausweg aus den politischen Konflikten zwischen monarchischen und demokratischen Legitimitätsansprüchen gesehen wird (Guizot 1985, S. 249), so führt auf Seiten des Marxismus die Verwissenschaftlichung der frühsozialistischen Planungsutopien zum Absterben des Staates: „An die Stelle der Regierung über Personen tritt die Verwaltung von Sachen und die Leitung von Produktionsprozessen“ (Engels 1973, S. 224). ${ }^{13}$ So zeigt sich, dass die Entwicklung des modernen, demokratischen Verfassungsstaates nicht nur durch einen normativen, an praktischer Autonomie orientiertem Politikbegriff geprägt ist, sondern zugleich von der - teils parallel verlaufenden, teils konkurrierenden - technisch geprägten Vorstellung gesellschaftlicher Steuerung begleitet wurde. Dieses technische Erbe des Politikbegriffs hat im zwanzigsten Jahrhundert dazu geführt, dass die technische Dimension rationaler Steuerung gänzlich gegen die vermeintliche Irrationalität des Politischen in Stellung gebracht werden konnte.

\section{Jenseits des Politischen: Die Gesellschaft als Skinner-Box}

Das utopische und postutopische Denken des neunzehnten Jahrhunderts spiegelt damit die Entwicklung jener rationalen Herrschaft, die Max Weber zu Beginn des zwanzigsten Jahrhunderts als Kernelement des modernen Anstaltsstaates ausmachte (Weber 2009). Zu diesem Zeitpunkt der Jahrhundertwende ist auch der Begriff der Technokratie erstmals geprägt worden. In den USA geht diese technokratische Perspektive zurück bis auf Woodrow Wilson und Frank Goodnow (vgl. Rosanvallon 2015, S. $90 \mathrm{ff}$.). Politik wird hier zur angewandten Verwaltungswissenschaft und dreht sich im wesentlich um die Frage der Zweck-Mittel-Rationalität. Im Mittelpunkt steht die Idee einer radikalen Effizienzsteigerung staatlicher Steuerungsleistungen: Angesichts der zunehmenden Komplexität moderner Gesellschaften erscheint politische Willensbildung und Entscheidung zunehmend irrtumsanfällig und

\footnotetext{
11 Vgl. zu den Texten Ramm (1968). Als Überblick über die Utopien des „technischen Staates“ im neunzehnten Jahrhundert Saage (2002).

12 Exemplarisch für dieses Denken ist Charles Fourier, der 1808 in seiner Theorie der vier Bewegungen mit Hilfe der ,exakten“ Wissenschaft, die ,in allen Punkten mit den Lehrsätzen der Geometrie“ übereinstimme, die ,philosophischen Wissenschaften zum Schweigen bringen“ will (Fourier 1966, S. 44 u. 57 ).

13 Dieses marxistische Ordnungsverständnis wurde dann allerdings auch wieder rückübersetzt in die utopische Textgattung: Edward Bellamy entwarf in den USA 1888 mit Looking Backward: 2000-1887 eine perfekt geordnete, betriebsförmig organisierte Industriegesellschaft, die weitgehend ohne staatliche oder politische Entscheidungsgewalt auskommt (Bellamy 1980).
} 
soll durch eine rationalisierte Form der Expertenherrschaft abgelöst werden (Meynaud 1964, S. 9). Staat und Gesellschaft sollten aus dieser Sichtweise so geführt werden, wie (männliche) Manager und Ingenieure die Abläufe der industriellen Produktion optimierten. ${ }^{14}$ Diese Vorstellungen sind im zwanzigsten Jahrhundert von ganz unterschiedlichen politischen Strömungen - vom Sozialismus über den Liberalismus bis hin zum Konservatismus - aufgegriffen und weiterentwickelt worden (Etzemüller 2009). Im europäischen Kontext wurde diese Idee exemplarisch von der sozial-demokratisch geprägten Steuerungsutopie Gunnar Myrdals verkörpert, in der die Frage der sozialen Verteilungsgerechtigkeit durch technisch-expertokratische Entscheidungsfindung rationalisiert werden sollte (Etzemüller 2010).

Das mit der Industrialisierung anbrechende Zeitalter der technologischen Hochmoderne (Scott 1999; Frauenholz et al. 2012) hat dieses fortschrittsgläubige Optimierungsdenken gegen Mitte des zwanzigsten Jahrhunderts fortgeführt. Der Verhaltenspsychologe B. F. Skinner knüpfte mit seinem einflussreichen Entwurf des behavioral engineering an die utopische Tradition an und definierte sie als applied psychology neu (Skinner 1953). Skinner greift dabei die Überlegungen auf, die bereits 1913 von John B. Watson entwickelt wurden. Dort war das Ziel des neuen verhaltenspsychologischen Forschungsansatzes sehr deutlich definiert worden: „Its theoretical goal is the predicition and control of behavior" (Watson 1913, S. 158).

Bemerkenswert an Skinners Position ist die Tatsache, dass er den Mechanismus der Verhaltensteuerung durch hierarchisch implementierte Anreizstrukturen in Form einer literarischen Utopie popularisiert hat und somit die Verbindung von wissenschaftlichem Erkenntnisgewinn und sozialem Ordnungsanspruch wieder deutlich sichtbar macht. ${ }^{15}$ Damit wiederholt sich hier ein klassisches Muster aus der politischen Ideengeschichte der Utopie: Naturwissenschaftliche Erkenntnisse und Paradigmen werden aus ihrem ursprünglichen Kontext herausgelöst und durch die Übertragung auf gesellschaftliche und politische Ordnungsfragen zum technischen Problemlösungswissen erklärt. In der Frühen Neuzeit waren es Astronomie und Geometrie, später kamen die Sozialmathematik (Condorcet) und die Sozialphysik (Quetelet) hinzu. Bei Skinner ist es nun die Sozialpsychologie, die das grundlegende Ordnungsproblem des menschlichen Zusammenlebens wissenschaftlich exakt lösen soll. Hinzu kommt, dass Skinners Utopie keineswegs nur eine akademische Fingerübung darstellte. Im Gegenteil: Wie Hilke Kuhlmann (2005) eindrücklich gezeigt hat, traf Skinners Walden Two in den USA auf eine zwar verspätete, aber dann seit etwa 1960 umso größere Resonanz und wurde in zahlreichen experimentellen Gemeinschaftsprojekten in die Tat umgesetzt.

Kurz nach dem Zweiten Weltkrieg entwarf Walden Two 1948 in Romanform ein fiktives Szenario, in dem auf der wissenschaftlichen Grundlage des Behavio-

\footnotetext{
${ }^{14}$ So forderte Thorstein Veblen die Einführung eines ,,practicable soviets of technicians“: ,,a suitable group of economists and engineers, who will have to be drawn together by self-selection on the basis of a common interest in productive efficiency, economical use of resources, and an equitable distribution of the consumable output“ (Veblen 1921, S. 152; vgl. zur Technokratiebewegung auch Willeke 1995).

15 Aus literaturwissenschaftlich-vergleichender Perspektive zu Walden Two Swirski (2011, 15ff.); aus diesem Grund steht in der Folge auch nicht die immanente Diskussion seiner Sozialpsychologie im Mittelpunkt (dazu vor allem Skinner 1953 und Skinner 1974), sondern die Fortschreibung der politischen Utopie mit Walden II.
} 
rismus eine perfektionierte Ordnung konstruiert wird. Unter der Führung des charismatischen Gründers Frazier obliegt einer kleinen Elite von sechs Planerinnen und Planern ${ }^{16}$ sowie einer Gruppe von ausführenden Managerinnen und Managern das verhaltenstechnische positive reinforcement, um die umfassende Bedürfnisbefriedigung und den sozialen Frieden zu sichern. Falsches Verhalten wird nicht durch Sanktionen verhindert, sondern das erwünschte Verhalten wird durch konditioniertes „Lernen“ gefördert. Die technokratische Pointe des Entwurfs liegt in der expliziten Distanz zu demokratisch verfasster, staatlicher Politik, die als traditionelle, aber ineffiziente Form harter Steuerung zugunsten der wissenschaftsbasierten weichen Steuerung verworfen wird. Allerdings ist damit auch die Abkehr vom Freiheitsbegriff verbunden: "If man is free, then a technology of behavior is impossible“ (Skinner 1976, S. 241; vgl. auch Skinner 1971b).

Damit präsentierte Skinner eine Ordnungsvorstellung, in der Staat und Regierung als Steuerungsinstanzen durch eine wissenschaftliche Verhaltensmodifikation abgelöst werden. Sowohl staatliche Regierung als auch demokratisches Entscheiden und die daraus resultierende rechtliche Ordnung sind aus dieser technokratischen Perspektive lediglich falsche Anreizsysteme zur Steigerung des Gemeinwohls, die ihr Ziel nur durch hierarchischen Zwang erreichbar machten.

Die wissenschaftsbasierte Konditionierung des Verhaltens setzt dagegen auf Steuerung, Kontrolle und Optimierung ohne direkte Herrschaftsausübung. Vielmehr geht es um das Design von Entscheidungskontexten und Rückkopplungsschleifen, die als weiche Macht eine sanftere, aber dafür auch nachhaltigere Wirkung entfalten sollen: Nicht die negative Sanktion durch den Gebrauch des Gewaltmonopols steht im Mittelpunkt der Aufmerksamkeit. Vielmehr sollen positive Anreizstrukturen geschaffen werden, mit denen das erwünschte Verhalten prämiert wird. Die Mitglieder dieser Ordnung werden demnach so konditioniert, dass ihnen die handlungsleitenden Ziele nicht wie eine Fremd-, sondern wie eine Selbststeuerung erscheinen. Damit wird das politische Gemeinwesen zur kollektiven Skinner-Box umgebaut jene konditionierende Versuchsanordnung, an der ihr Namensgeber mit Hilfe von Labormäusen die Techniken der positiven Verhaltensverstärkung zuerst erprobt hatte. ${ }^{17}$

Die Abkehr vom demokratischen Verfassungsstaat ist dabei nur vordergründig als ein Bruch mit der utopischen Tradition zu verstehen. Seit Morus hatte das Genre immer schon Platons Wissensherrschaft zur Grundlage einer rationalisierten Entscheidungsstruktur gemacht, die sich politisches Handeln nicht mehr als ein Ausdruck von selbstbestimmter und ergebnisoffener Freiheit vorstellen mochte, sondern vielmehr als eine instrumentelle Form zur Durchsetzung der objektiv besten Ergebnisse. Zum gleichen Zeitpunkt also, an dem die Orwellsche Dystopie den utopischen Diskurs abgelöst und die totalitäre Dimension der humantechnologischen Zentralsteuerung

\footnotetext{
16 „There are six Planners, usually three men and three women. The sexes are on such equal terms here that no one guards equality very jealously“ (Skinner 1976, S. 48). Kritisch zu den Grenzen dieses Gleichheitsanspruches Wolpert (2005).

17 Durch die nicht aufgehobene Differenz von Planenden und Planungsunterworfenen unterscheidet sich Skinners Entwurf auch im Grundsatz von entscheidungstheoretisch begründeten Formen konstitutioneller Selbstbindung, wie sie beispielsweise bei Elster 2000, 88 ff. diskutiert werden.
} 
offengelegt hatte, kehrt Skinner zurück zu einer Vorstellung der grundlegenden Planbarkeit sozialer Ordnung. Skinners Utopie wirkt dabei in Richtung einer radikalen Enthistorisierung: Die Zukunft rückt noch näher an die Gegenwart, die moderne Gesellschaft besitzt in Gestalt der avancierten Verhaltenswissenschaften die Mittel zur Verwirklichung ihrer eigenen Zukunft im Hier und Jetzt.

Gleichwohl bleibt auch in dieser antistaatlichen Perspektive das zentrale Ziel die Erzeugung kollektiver Sicherheit. Die Konditionierung des ,behavioral engineering" (Skinner 1976, S. 93) dient der wissenschaftlichen Minimierung aggressiven Verhaltens. ${ }^{18}$ Der Staat erscheint jedoch für diesen Zweck als ein überholtes Instrument: „A state defined by repressive, formal, legal, social controls based on physical force is not necessary in the development of civilization, and although such a state has certainly figured in our own development, we may be ready to move on to another stage“" (Skinner 1976, S. xv).

Skinner wählt dagegen das Vorbild von Henry David Thoreaus Walden für seine eigene Utopie: Mitte des neunzehnten Jahrhunderts hatte sich Thoreau aus der Gesellschaft in eine selbstgebaute Blockhütte in den Wäldern Massachusetts zurückgezogen, um im Einklang mit der Natur Widerstand gegen die Eingriffe in seine individuelle Selbstbestimmung zu leisten. Sein literarischer Erfahrungsbericht wurde zu einem überaus einflussreichen Bestseller, dessen folgende Rezeption durch die Entwicklung zur technisch-industriellen Zivilisation noch verstärkt wurde. Thoreaus radikalisierte Autonomie hatte jedoch den Nachteil, dass Autarkie und Herrschaftsfreiheit lediglich in Form eines sozialen Solipsismus zu verwirklichen waren. Die Herausforderung für Walden II lag demnach darin, dieses Ideal auch für eine soziale Gemeinschaft auf der Höhe des wissenschaftlich Machbaren zu ermöglichen.

Die Fortschritte in der psychologischen Steuerungstechnik versprechen für Skinner nun genau diese Schwäche von Walden I zu überwinden und eine soziale Ordnung zu errichten, in der ein Leben im Einklang mit den natürlichen Bedürfnissen auch in Gemeinschaft realisierbar ist. ${ }^{19}$ Dazu ist jedoch nicht nur eine Abkehr von den Irrwegen staatlicher Lösungen notwendig. Auch die Beschäftigung mit der Geschichte hat für die Suche nach der Lösung gesellschaftlicher Probleme ausgedient. Historisches Erfahrungswissen wird angesichts der neuen, experimentell gewonnenen wissenschaftlichen Möglichkeiten radikal entwertet. Bereits in Aldous Huxleys Brave New World zitiert das Weltregierungsmitglied Mustapha Mond den als säkularen Heiligen verehrten Henry Ford mit seinem Satz ,history is bunk“ (Huxley 1977, S. 38). Auch in Walden II hat Geschichte nur noch zum Zweck der Unterhaltung einen Platz. ${ }^{20}$

\footnotetext{
${ }_{18}$ Die deutsche Übersetzung von Walden Two fügt den Untertitel „Die Vision einer aggressionsfreien Gesellschaft“ hinzu (Skinner 1971a). Der deutsche Titel „Futurum zwei“ entbehrt zudem nicht einer gewissen Ironie: Das ,wird gewesen sein“ fügt sich bestens in die von Reinhart Koselleck untersuchte Semantik der „vergangenen Zukunft“ (Koselleck 1979) ein - inzwischen ist Skinners hochmoderner Futurismus in deutscher Sprache nur noch antiquarisch erhältlich.

19 Rezensionen des Werkes haben auf die Ähnlichkeit zwischen der Walden II-Kolonie und puritanischen Kleinstädten wie Skinners Geburtsort Susquehanna in Pennsylvania hingewiesen (Der SPIEGEL, 19.10.1981, https://www.spiegel.de/spiegel/print/d-14339943.html).

20 „History is honored in Walden Two only as entertainement. It isn't taken seriously as food for thought“ (Skinner 1976, S. 106). Vergleichend zu Skinner, Huxley und Orwell (Krause 2007).
} 
Welche Folgen hat nun diese Abkehr von der Tradition des demokratischen Verfassungsstaats für die Gestaltung der neuen Ordnung? Zunächst ist auffällig, dass mit dem literarischen Manifest der behavioristischen Verfassung einerseits offen für dieses Modell geworben wird. ${ }^{21}$ Andererseits jedoch ist eines der Kernmerkmale, dass für die Bewohnerinnen und Bewohner der neuen Gemeinschaften deren Struktur selbst weitgehend verborgen bleibt. Eine Öffentlichkeit ist nicht notwendig und würde die Rationalität der planerischen Entscheidungen nur beeinträchtigen: ,We deliberately conceal the planning and managerial machinery to further the same end. I doubt whether there are half a dozen members, aside from the Managers, who can correctly name all six Planners“ (Skinner 1976, S. 220).

Deutlich wird diese Abkehr von zentralen Grundprinzipien liberaler Demokratien auch in der Frage nach dem Gründungsakt. Auch dieser muss in Walden II arkan bleiben, weil ein öffentliches Bewusstsein der eigenen Gründung die Historizität des Gemeinwesens sichtbar machen würde: „For the same reason [...] we discourage any sense of history. The founding of Walden two is never recalled publicly by anyone who took part in it“" (Skinner 1976, S. 221). Die historische Genealogie bedroht damit den rationale Geltungsanspruch und wird radikal aus dem Bewusstsein der Mitglieder verdrängt. Trotz ihres technischen Kerns darf der gemachte Charakter der Ordnung nicht sichtbar werden. Der republikanische Aspekt der Erfahrungsgebundenheit politischer Ordnung, der in eine erhöhte Bedeutung historischer Narrative übertragen wird, ist in der technokratischen Ordnung in sein Gegenteil verkehrt: Technische Rationalität kann ihre eigene Historizität nicht abbilden, ohne den eigenen Geltungsanspruch des Innovativen damit zu unterlaufen. Technische Innovation lebt daher von der neuzeitlichen Überzeugung, dass das Neue dem Alten aufgrund des zwangsläufig damit verbundenen Wissensvorsprunges überlegen sein muss. ${ }^{22}$ Technik von gestern ist damit nicht Gegenstand normativ sinnvoller Erfahrung, sondern lediglich veraltet. Dass die Erinnerung an Ursprünge, Prototypen und erste Serienmodelle sowie die Ausstattung von Produkten mit einer Geschichte unter den Gesichtspunkten des Produktmarketing erfolgreich sein kann, ist bereits eine postmoderne Brechung der Dominanz zukunftsorientierter Innovation, wie sie die technische Hochmoderne geprägt hat. ${ }^{23}$

Skinners Theorie des ,positive reinforcement“" versteht die Gründung eines Gemeinwesens daher vielmehr als eine experimentelle Versuchsanordnung, die je nach Resultat immer wieder neu optimiert werden kann und auf diese Weise mit der

\footnotetext{
${ }^{21}$ Allerdings nicht mit den erwünschten Folgen: Die realen Versuche, Walden II in die Tat umzusetzen, scheiterten allesamt. Die Sozialexperimente auf der Grundlage von Skinners Utopie zogen zu viele Personen an, die sich in der Rolle der Führenden gesehen haben und zu wenige, die sich gerne hätten führen lassen: „most of them tacitly assumed that they would be the Frazier of their group“ (Kuhlmann 2005, S. xii).

${ }^{22}$ Damit illustriert Skinners demiurgisches Modell auf originelle Weise die Diagnose der spezifisch neuzeitlich-cartesianischen, ahistorischen „Selbstauffassung der Vernunft als des Organs eines absoluten Anfangs [...]. Der absolute Anfang, der Geschichte inauguriert, verbietet sich selbst, Geschichte zu haben“" (Blumenberg 1996, S. 159).

23 „Während der Progressismus in den Jahrzehnten nach dem Zweiten Weltkrieg auf die Entwertung der Vergangenheit [...] ausgerichtet war, ging der in diesem Zeitraum entstehende neue Progressismus mit einer Rehabilitierung der Vergangenheit einher, deren Aufwertung als eine der Denk- und Möglichkeitsbedingungen von Zukunft betrachtet wurde" (Boltanski und Esquerre 2018, S. 105).
} 
Zeit immer effizienter wird, ohne dass die Zeitlichkeit dieser Ordnung zum Teil der öffentlichen Selbstbeschreibung wird. Maßgeblich ist die Herstellung adaptierter Entscheidungsanreize, mit der die Planer und Manager der Ordnung regelmäßig ein Update verpassen und damit den Kontrollmechanismus justieren. ${ }^{24}$

Damit, und dies ist im Grunde das Hauptargument Skinners gegen die traditionelle Begründung von Staat und Recht, wird der strafende Sanktionsmechanismus zur Vermeidung negativer Handlungen durch einen positiven Verstärkermechanismus von erwünschten Handlungen ersetzt. Wichtig für Skinner ist es dabei, zu betonen, dass der behavioristische Kontrollanspruch nicht direkt auf das Verhalten selbst gerichtet ist, sondern indirekt auf der motivationalen Ebene der Wünsche und Wertvorstellungen wirksam wird: ,we control not the final behaviour, but the inclination to behave - the motives, the desires, the wishes" (Skinner 1976, S. 246f.). Auch hier ist eine bemerkenswerte Parallele zur gut zehn Jahre zuvor erschienen Dystopie von Huxley zu beobachten: Dessen Hauptfigur Helmholtz Watson arbeitet am „College of Emotional Engineering“ und die Konditionierung der Leidenschaften ist eine der zentralen Herrschaftstechniken in Brave New World. Skinner und Huxley stellen sich - unter umgekehrten normativen Vorzeichen - die moderne Utopie als eine governance der Affekte vor, wie sie in der utopischen Tradition schon bei Charles Fourier in der Theorie der vier Bewegungen im Zentrum steht und die bereits im achtzehnten Jahrhundert in der Aufklärungstheorie thematisiert wurde (Fourier 1966; Hirschman 1977).

Genau in dieser Affektökonomie liegt damit auch der Übersprung von der wissenschaftlichen Beobachtung äußeren Verhaltens zu Kontrollansprüchen innerer Zustände und Einstellungen des beobachteten Subjektes, mit dem letztlich eine wesentliche Selbstbegrenzung des Liberalismus aufgegeben wird (Fischer 2014). ${ }^{25}$ Der Liberalismus hatte immer auch den Anspruch, liberale Subjekte über Bildung und über die Sozialisierungsleistung liberaler Institutionen zu formen. Darin lag das republikanische Erbe, politische Ordnung nicht nur auf äußerliche Verhaltensdispositionen, sondern auch auf die motivationalen und ethischen Überzeugungen der Bürgerinnen und Bürger zu legen (Schulz 2015). Dieser latente Subjektivierungsanspruch liberalen Denkens wird nun in der Hochmoderne an neuere, vermeintlich effizientere Steuerungstechniken gekoppelt und kehrt sich mit seinen technokratischen Konsequenzen gegen den Liberalismus und seine normativen Grundannahmen von Freiheit und Autonomie.

Freiheit und Autonomie sind für Skinner jedoch nur verdeckte Chiffren einer planlosen Gesellschaft, die Kontingenz und Willkür anstelle von Rationalität und verbindlichen Zielen den Vorzug gibt. Angesichts der irrationalen Kollateralschäden des liberalen Freiheitsverständnisses ist für Skinner klar: „there's no alternative to a planned society.“ (Skinner 1976, S. 248). Die hier an den Liberalismus gerichtete

\footnotetext{
${ }^{24}$ „If it's in our power to create any of the situations which a person likes or remove any situation he doesn't like, we can control his behaviour. When he behaves as we want him to behave, we simply create a situation he likes, or remove one he doesn't like. As a result, the probability that he will behave that way again goes up, which is what we want. Technically it's called ,positive reinforcement “ ' (Skinner 1976, S. 244).

${ }^{25}$ Zur Diskussion der Affektökonomie in der digitalen Gesellschaft vgl. Mühlhoff et al. (2019), zum Verständnis des Internets als „Affektmaschine“ Reckwitz (2017, S. 234).
} 
Kritik trifft im Übrigen auch die Demokratie. Hier nimmt Skinner Argumente des klassischen Liberalismus auf, die den normativen Begriff von politischer Teilhabe empirisch kritisieren. Das funktionalistische Argument, demokratische Partizipationsversprechen seien angesichts der geringen individuellen Aussichten auf Beeinflussung des Wahlausgangs hinfällig (Skinner 1976, S. 249), hatte bereits Benjamin Constant in seiner Überlegung zur Freiheit der Alten und der Modernen gebraucht (Constant 1972). Der Despotie-Vorwurf (prominent Chomsky 1959, 1971; Koestler 1967) wurde von Skinner vorhergesehen und mit Verweis auf die Despotie der Mehrheit abgewehrt, die ebenso wie die Despotie der Ignoranz und die Despotie des Zufalls der wahre Feind einer guten Ordnung sei (Skinner 1976, S. 252). Mitbestimmung ist in Walden II allein als technische feedback-Schleife eingeplant: „The government of Walden two [...] has the virtues of democracy but none of the defects. [...] Every Member has a direct channel through which he may protest to the Managers or even the Planners. And these protests are taken seriously as the pilot of an airplane takes a sputtering engine. We don't need laws and a police force to compel a pilot pay attention to a defective engine. [...] Similarly, our Behavioral Cultural Managers need not be compelled to consider grievances. A grievance is a wheel to be oiled, or a broken pipeline to be repaired“ (Skinner 1976, S. 253). Entscheidend ist damit primär eine empirische Frage der technischen Effizienz, nicht jedoch eine normative Frage der Autonomie. Die Verfassung und die Regierung von Walden II ist folgerichtig Sache von Spezialistinnen und Spezialisten. Verfassungsänderungen bedürfen des einstimmigen Votums der Planerinnen und Planer und zwei Drittel der Managerinnen und Manager (Skinner 1976, S. 254). Für die rationale Effizienz wird damit der normative Kern des Liberalismus geopfert.

Skinners Behaviorismus markiert eine Verschiebung in der Beschreibungssemantik moderner Gesellschaften. Die durch die philosophische Tradition des Liberalismus geprägte Sprache von Freiheit und Individualität ist für den Behaviorismus keine korrekte Abbildung der sozialen Wirklichkeit und damit falsch. Die eigene, naturwissenschaftlich codierte Semantik hingegen wird als objektive Beschreibung der Gesellschaft präsentiert. Der entscheidende Unterschied zwischen Naturbeobachtung und gesellschaftlicher Selbstbeschreibung liegt jedoch darin, dass erstere ihren Gegenstand nur beschreibt, um ihn besser zu beherrschen - letztere aber mit ihrer Semantik immer auch die Geltung gesellschaftlicher Normen und Handlungsoptionen konstruiert. Wie im dritten Abschnitt gezeigt werden soll, spielt genau dieser semantische Konflikt aber auch in der Digitalisierungsdebatte eine wichtige Rolle, die damit das uneingestandene Erbe der technischen Steuerungsutopien antritt.

\section{Social engineering in der digitalen Konstellation}

Skinners szientifisches Verständnis von Politik und Gesellschaft in der technokratischen Hochmoderne ist nicht ohne Widerspruch geblieben. Die fünfziger und sechziger Jahre haben eine ganze Reihe von technikkritischen Positionen hervorgebracht, die sich sowohl gegen den Behaviorismus im Besonderen als auch gegen die zunehmende Technisierung des Politischen im Allgemeinen richteten (Ellul 1964; Anders 
1956; Mumford 1977). ${ }^{26}$ Diese kulturkritisch ausgreifenden Diagnosen werden 1961 von Helmuth Schelsky in seinem Essay Der Mensch in der wissenschaftlichen Zivilisation zur politischen Herausforderung des ,technischen Staates“ verdichtet, in der auf die umfassende Technisierung der Gesellschaft nur mit einer technokratischen Transformation des Staates geantwortet werden kann, wenn ein Anspruch auf politische Steuerung überhaupt noch aufrechterhalten werden soll (Schelsky 1979, S. 468 ff.). Der technokratische Ordnungsentwurf des Behaviorismus blieb auch in der politischen Theorie nicht unwidersprochen. Während die amerikanische Politikwissenschaft schon früh den naturwissenschaftlichen Erklärungsmodellen nacheiferte und sich der modifizierte Ansatz des Behavioralismus mit einem erweiterten Verhaltensbegriff und abgeschwächtem Steuerungsanspruch durchsetzte, ${ }^{27}$ ging die normativ orientierte Theoriediskussion zu dieser technokratischen Erblast der social sciences auf Distanz und versuchte, an den von Aristoteles und dem deutschen Idealismus geprägten politischen Handlungsbegriff anzuknüpfen (Arendt 1981; Taylor 1964; Habermas 1969).

Ungeachtet dieser massiven Kritik an der Technisierung von Politik und Gesellschaft setzte sich die utopische Steuerungshoffnung sowohl im Kontext der amerikanischen Alternativkultur (Turner 2006) als auch im staatlichen und ökonomischen Bereich fort (Lepore 2020). Der letzte Abschnitt des Beitrages wird daher exemplarisch die Kontinuität der technischen Steuerungserwartungen und ihre politische Relevanz aufzeigen. Dabei ist die mit dem Computer und der Digitalisierung verbundene Umbruch sehr viel weniger ein revolutionärer Einschnitt, als eine Fortsetzung von strukturellen Mustern der Technisierung und der Maschinisierung - insofern haben Computer vielmehr dazu beigetragen, diese Entwicklung zu stabilisieren als sie zu transformieren (Weizenbaum 1977, S. 54f.; mit einer vergleichbaren These auch Nassehi 2019). Zuletzt hat Zuboff gezeigt, wie sehr die Steuerungserwartungen von Skinner die digitalen Gegenwartsprojekte geprägt haben (Zuboff 2018, S. 377). Diese Debatten bilden damit einen wichtigen Vorgriff auf die digitale Konstellation. Wissenschaftsgeschichtlich ist dieser Einflussprozess jedoch durch den Umbruch von der behavioristischen Sozialpsychologie zur Verhaltensökonomie markiert. Die vom Behaviorismus prognostizierten Möglichkeiten der Verhaltenssteuerung werden jetzt durch eine Verbindung von Verhaltensökonomie und Big Data erwartet. Damit wiederholt sich mit der „californian ideology“ (Barbrook und Cameron 1995) ein Muster in der Entwicklung der klassischen Utopien, die sich stets von der Entwicklung neuer Wissensformen radikal neue Steuerungsmöglichkeiten auf dem Weg zur perfekten Ordnung erhofften.

Ein besonders prominentes Beispiel ist die Diskussion um das „Nudging“, das als weiche Form der Steuerung die paradoxe Verbindung von libertärer Entscheidungsfreiheit und normativ geleiteter Beeinflussung der outcomes ermöglichen soll: Cass Sunstein und Richard Thaler haben mit ihrer Theorie den Versuch unternommen, die alte Frage der Gemeinwohlorientierung politischen Handelns in die verhaltenstheo-

\footnotetext{
26 Zur direkten Anknüpfung an Anders Technikkritik im Kontext der Digitalisierung vgl. Nosthoff und Maschewski (2019).

27 So u. a. bei David Easton, Karl Deutsch, Gabriel Almond und Sidney Verba (vgl. Falter 2019); zum Steuerungsanspruch in der Politikwissenschaft Görlitz und Burth (1998).
} 
retische Konstruktion von Entscheidungsarchitekturen zu überführen (Thaler und Sunstein 2009). In der Sozialwissenschaft hat sich damit zunächst unabhängig von der Digitalisierungsdebatte eine Fortsetzung der Steuerungshoffnung entwickelt, die im Wesentlichen seit den siebziger Jahren aus der Kombination der sozialpsychologischen Ansätze mit Verhaltensökonomie und der Frage nach der Rationalität von Konsumentscheidungen erwachsen ist (Bröckling 2017, S. $182 \mathrm{ff}$.). ${ }^{28}$ Der von Sunstein und Thaler propagierte ,libertäre Paternalismus“ (Thaler und Sunstein 2003) zielt auf eine weiche Steuerung, bei der die Präsentation von Entscheidungsalternativen für die Konsequenzen des tatsächlichen Entscheidens eine große Rolle spielt. Die individuelle Entscheidungsfreiheit soll demnach normativ erhalten bleiben, die faktische Entscheidungswahrscheinlichkeit aber durch die Gestaltung der äußeren Entscheidungskontexte maßgeblich beeinflusst werden.

Das Besondere der Verhaltensökonomie im digitalen Zeitalter besteht nun in der Möglichkeit, die Beeinflussungsmechanismen zunehmend zu personalisieren und damit ihre effektive Wirksamkeit weiter zu erhöhen (Sunstein 2015, S. 155 ff.; Pohle et al. 2018, S. 13 ff.; Stalder 2016, S. 228 f.). Dieser Ansatz hat naheliegend nicht nur privatwirtschaftliche Anwendung, sondern wird auch von staatlichen Regierungen genutzt, um gemeinwohldienliche outcomes ohne direkten Einsatz von gesetzlichem oder finanziellem Zwang zu erreichen. ${ }^{29}$ Kritik findet die Steuerung des Nudging prominent durch den Gouvernementalitäts-Ansatz, der diese weiche Form der Steuerung immer schon in das Dispositiv technisch-instrumenteller Machtstrukturen eingeordnet hatte. Während in Skinners Utopie der Verhaltenssteuerung die Expertinnen und Experten noch einen personalen Einfluss auf die Steuerung ausüben mussten, so kehrt im algorithmengestützten Optionsdesign „,der gute Hirte als Maschinenutopie“ zurück (Bröckling 2017, S. 193). Dabei wird auch auf einen blinden Fleck verwiesen, der in den Ansätzen der Verhaltensökonomik zumeist unterschlagen wird: Verhaltenssteuerung findet in der sozialen Wirklichkeit nicht unter Laborbedingungen statt, sondern steht in einem permanenten Konkurrenzverhältnis unterschiedlicher Steuerungsanreize einer Vielzahl von Akteuren - seien es staatliche Institutionen oder privatwirtschaftliche Unternehmen (ebd.). Hinzu kommt, dass bei der Umsetzung verhaltenswissenschaftlicher Policy-Empfehlungen im politischen Entscheidungsprozess eine weitere Reduktion von Komplexität stattfindet: Das politisch verwendete Wissen entspricht selten der verhaltenswissenschaftlichen Erkenntnis, sondern

\footnotetext{
28 Die sozialtechnischen Steuerungsansprüche erinnern stark an das behavioristische Paradigma - andererseits taucht Skinner aber im libertären Paternalismus und auch in der Verhaltensökonomie als Referenz nicht auf. Der Grund hierfür ist, dass Nudging auf cognitive science, einem Zweig der Verhaltenswissenschaften beruht, der sich explizit von Skinners und Watsons behaviorism abwendet. Allerdings bleibt das neue Paradigma der Kognitionswissenschaft in seiner Abwendung von der behavioristischen Sozialpsychologie ambivalent: Kognitionspsychologie ist so ,defined by the aim to open the mind's black box and to investigate how its different constituents such as memory, cognition, and perception interact to produce behavior. As such, it was directed explicitly against the behaviorism of Watson and Skinner, although it was in line with the behavioralist or engineering approach of twentieth-century U.S. psychology“ (Heukelom 2014, S. 97).

29 Einen umfassenden Überblick über die Diskussion und die Anwendungsbereiche von Nudging im Zusammenspiel mit Big Data bietet das Gutachten von Pohle et al. (2018); zur problematischen Unterwanderung des normativen, mit der rule of law verbundenen Geltungsanspruches durch das primär instrumentelle Nudging vgl. Lepenies und Malecka (2015).
} 
instrumentalisiert diese im Sinne politischer Interessendurchsetzungen. ${ }^{30}$ Auf diese Weise verkehrt sich die rationale Steuerung auch unter digitalen Bedingungen in den Kampf um die stärkste Steuerungsmacht. ${ }^{31}$

Nochmals radikalisiert - und sehr viel weniger auf die politischen als auf die privatwirtschaftlichen Steuerungsansprüche ausgerichtet - wird das Modell der Verhaltenssteuerung unter dem sprechenden Begriff der Sozialphysik. Dabei handelt es sich um den Versuch des MIT-Informatikers Alex Pentland, mithilfe von Big Data die Lösung sozialer Distributions- und Steuerungsprobleme ohne staatliche Institutionen in Angriff zu nehmen und damit das Konditionierungsmodell von Skinner in das digitale Zeitalter zu übertragen (vgl. Zuboff 2018, S. 481 ff.). Der Anspruch dieses Modells ist es, die verhaltensökonomisch effiziente Steuerung von Kleingruppen in Unternehmen auf die Gestaltung von sozialen und politischen Einheiten zu übertragen und damit wie Skinner eine Alternative zu den als zu rigide empfundenen Steuerungsformen durch Politik und Gesetze anzubieten: „Social physics is a quantitative social science that describes reliable, mathematical connections between information and idea flow on the one hand and people's behaviour on the other. [...] It enables us to predict the productivity of small groups, of departments within companies, and even of entire cities. It also helps us tune communication networks so that we can reliably make better decisions and become more productive“" (Pentland 2015, S. 4).

Big Data dient in diesem Ansatz dazu, das social engineering von der Theorieund Modellbildung weitestgehend unabhängig zu machen - mit Hilfe der Informationsverarbeitung und der Datafizierung größerer sozialer Einheiten ist es nun möglich, den Gegensatz von Laborexperimenten und sozialer Wirklichkeit zu überwinden und die soziale Wirklichkeit selbst zur kybernetischen Datenquelle zu machen. Big Data als ,,reality mining“ operiert daher im Kontext von ,living laboratories“ - Gesellschaften werden auf diese Weise zum Gegenstand einer umfassenden Vermessung über lange Zeiträume hinweg und können nicht mehr nur unter den eingeschränkten experimentellen Laborbedingungen, sondern in Echtzeit direkt beobachtet werden: Nach der Erfindung des Teleskops und des Mikroskops kann jetzt das digitale „socioscope“ zum Einsatz kommen und Einblick in die Gesetzmäßigkeiten der bislang opaken sozialen Praxis liefern (Pentland 2015, S. 10). Damit bringt Pentland deutlich zum Ausdruck, wie sehr die digitalen Möglichkeiten der Informationsverarbeitung und -verknüpfung dem Traum einer durchweg rationalen

\footnotetext{
30 Diese Wissen repräsentiert daher eher,,a view of scientific knowledge about behaviour that proponents of behavioural policy would wish to have in order to change individual behaviours in a way they imagine“" (Malecka 2021).

31 Hinzu kommt, dass die institutionelle Umsetzung keineswegs ohne unmittelbare Machteffekte auf die Individuen ist, wie es die Nudgingtheorie behauptet: Am Beispiel des britischen Einsatzes von psychometrischen Tests für die Inanspruchnahme staatlicher Dienste und daraus resultierender Subjektivierungseffekte Crombin und Willis (2014).
} 
und effizient steuerbaren gesellschaftlichen Ordnungsstruktur entgegenzukommen scheinen. $^{32}$

Pentlands Ansatz ist - ebenso wie bei Skinner - auf soziale Lernprozesse gerichtet: Die Nachahmung von Gewohnheiten und Normen bildet die Grundlage für „crowd wisdom“ (Pentland 2015, S. 29). Indem ideale Interaktions- und Kommunikationsbedingungen geschaffen werden, sollen diese Nachahmungsprozesse für die Steigerung der sozialen Produktivität nutzbar gemacht werden. Auf diese Weise soll es dann gelingen, nützliches Wissen und Ideen möglichst schnell und effizient in einer Gruppe zu verbreiten (Pentland 2015, S. 34). Dabei setzt Pentland vor allem auf den Mechanismus des sozialen Anpassungsdrucks: „Seeing members of our peer groups adopting a new idea provides a very strong motivation to join in and cooperate with others" (Pentland 2015, S. 65).

Während damit vor allem Anwendungen in der Produktivitätssteigerung von Unternehmen beabsichtigt sind, so zeigt sich der politische Geltungsanspruch vor allem an der Übertragung des verhaltenssteuernden Imitationsmodells auf ganze Städte. Pentlands Ziel sind die Smart Cities: Seine Theorie dient dazu, Städte in ,data-rich, dynamic, responsive organisms“ zu verwandeln (Pentland 2015, S. 153). Das analoge Walden II wird damit zur vergangenen Zukunft - die verdateten Städte von morgen sind hingegen digitale Ideenmaschinen, deren Parameter eine ungeahnte Steigerung von Effizienz, Produktivität und Innovationen ermöglichen. Die digitalisierte Sozialphysik erlaubt es durch die Entdeckung von Mustern und Regelmäßigkeiten, als regulatives Vorhersageinstrument zu dienen (Pentland 2015, S. 191). Dazu aber, und damit steht Pentland ganz in der Tradition des naturwissenschaftlichen Objektivierungsparadigmas, bedarf es einer grundsätzlich anderen Beschreibungssprache als es uns die traditionellen Begriffe von Gesellschaft nahelegen: „I believe that its ultimate impact also depends upon whether it provides people [...] a language that is better than the older vocabulary of markets and classes, capital and production. [...] In this book I will put forward a new set of concepts with which I believe we can more accurately discuss our world and plan the future" (Pentland 2015, S. 8).

Diese beiden exemplarischen Ansätze des Nudging und der Sozialphysik lassen deutlich erkennen, wie sehr der Solutionismus des digitalen Tech-Zeitalters das Erbe früherer technokratisch geprägter Vorstellungen fortsetzt (Mozorov (2013; Strohschneider 2014). Die vermeintliche, digital erzeugte Verfügbarkeit und Beherrschbarkeit, die mit einer oberflächlichen Übersetzung sozialer und politischer Herausforderungen in technisch lösbare Probleme angestrebt wird, erfolgt jedoch um den hohen Preis einer semantischen Verarmung gesellschaftlicher Selbstbeschreibung. In seiner eindimensionalen Radikalität hat die Sozialphysik von Pentland das Problem unwillkürlich auf den Punkt gebracht: Die digitale Konstellation hat in den liberalen Demokratien einen Kampf um Deutungsmacht wieder zum Vorschein gebracht, der

\footnotetext{
32 Dabei werden solche Konzepte in Form des ,demos scraping“ auch von Regierungen eingesetzt, um durch rationale Kenntnis der Bedürfnisse des Demos rationale Entscheidungen herstellen zu können (für eine kritische Diskussion der technokratischen Schlagseite Ulbricht 2020) - damit schreibt dieses digitale Instrument unter Umgehung konfliktträchtiger demokratischer Verfahren der Willensbildung den von Guizot gehegten Traum einer ,Souveränität der Vernunft“ fort (Guizot 1985). Warum ,,algorithmic governance" trotz hoher Responsivität ein Problem für die Legitimität demokratischer Entscheidungen darstellt, zeigt König 2018.
} 
bereits in der klassischen Hochmoderne nach dem Zweiten Weltkrieg geführt worden ist und der nicht zuletzt eine Auseinandersetzung über die angemessene Beschreibungssprache gesellschaftlicher und politischer Ordnung darstellte. Seit Thomas Hobbes dem aristotelischen Paradigma des Politischen den Kampf angesagt hatte (Skinner 1996), ist dieser Konflikt bis in das zwanzigste Jahrhundert hinein ausgefochten worden. Auch in der Frage nach der Bedeutung des Digitalen für Politik und Gesellschaft taucht dieser Konflikt in verschärfter Form wieder auf. Zu rechnen ist daher im gegenwärtigen Lichte technischer Optimierungsversprechen mit einem verschärften Druck technokratischer Beschreibungssemantiken auf die Prozesse der gesellschaftlichen Willens- und Entscheidungsfindung.

Welche Konsequenzen ergeben sich also aus diesem Kampf um Deutungsmacht für die Zukunft des demokratischen Verfassungsstaates? Die Erkenntnis der ideengeschichtlichen Wurzeln der digitalen Konstellation schützt keineswegs gegen ihre zukünftigen Folgen. Wenn es dem Konditionierungskalkül, welches ,das Valley Denken nennt“" (Daub 2020), gelingt, tiefer in die normativen Selbstbeschreibungsdiskurse der liberalen Demokratie einzudringen und das technokratische Verständnis kollektiver Entscheidungsfindung weiter zu festigen, dann wird im Gebrauch der technisch bereitgestellten Möglichkeiten auch immer weniger verständlich sein, warum in liberalen Demokratien nicht in gleichem Ausmaße die sicherheits- und effizienzstiftenden Kontrollpotentiale des Digitalen ausgereizt werden sollten, wie dies in autokratischen Ordnungen gerade erprobt wird.

Eine weitere gewichtige Folge der technischen Entwicklung dürfte das sich verändernde Verhältnis zur Zukunft sein. Digital gestützte Verhaltensvorhersagen - predictive analytics - drohen paradoxerweise jene Zukunft abzuschaffen, aus deren Verheißungen sie im Kontext des liberalen Fortschrittsdenkens überhaupt erst entstanden sind (Loewenstein 2015). Die Zukunft scheint damit zusehends den offenen Charakter eines Reichs der Möglichkeiten zu verlieren und zu einem geschlossenen Raum, eine bruchlose Extrapolation der Gegenwart zu werden, in der jedes Subjekt im ewigen Jetzt der eigenen Vorlieben und Verhaltensdispositionen eingefroren wäre (Bridle 2019)..$^{33}$ Über die technologisch verfügbar gemachte Selbsttransparenz wird zwar eine erhöhte Gewissheit eigener Erwartungen und Präferenzen hergestellt. Mithilfe dieser steuerungsaffinen Instrumente aber berauben sich liberale Gesellschaften möglicherweise auch des offenen Horizontes einer nicht verfügbar gestellten Zukunft. Trotz aller Disruptionsrhetoriken der schumpeterianischen techno-entrepreneurs gilt auch für die Silicon Utopias, was Ralf Dahrendorf zum Kennzeichen von Utopien schlechthin machte: ,Alle Utopien von Platons Staat bis zu George Orwells schöner neuer Welt von 1984 haben ein Konstruktionselement gemeinsam: sie sind sämtlich Gesellschaften, in denen der Wandel fehlt“" (Dahrendorf 1965, S. 85).

33 Optimistischer zur demokratietheoretischen Kompatibilität von Kontingenz und KI hingegen Koster (2021). 


\section{Schlussbetrachtungen und Ausblick}

Der Beitrag hat gezeigt, wie sehr die mit der Digitalisierung verknüpften politischen Ordnungsvorstellungen auf eine lange Tradition technischer Steuerungsutopien der Moderne zurückgehen. Damit wird eine historische Genealogie sichtbar, die den eigenen, mit der Digitalisierung erhobenen Geltungsanspruch des radikal Neuen in mehrfacher Hinsicht relativiert. Ein solchermaßen weitgespannter Bezugshorizont kann an dieser Stelle kaum alle relevanten Texte und Bezüge abdecken und muss in seiner exemplarischen Auswahl zwangsläufig Lücken lassen. Daher sind hier nur die einschlägigsten und bekanntesten Beispiele der politischen Utopiegeschichte herangezogen worden. Gleichwohl sollte damit ein neuer und kontextualisierender Blick für die politiktheoretische Einordnung der Digitalisierung eröffnet worden sein. Auch hier bestehen über die genannten Verbindungen hinaus zahlreiche weitere Möglichkeiten, die Diskussion über die angemessene Gegenwartsdiagnostik weiter zu bereichern. Insbesondere die technisierungskritische Diskussion der fünfziger bis siebziger Jahre verdient es, im Detail aufgearbeitet zu werden. Inwieweit die politiktheoretische Kritik von Arendt, Taylor und Habermas an Behaviorismus und Technokratie eine tragfähige Perspektive für die politiktheoretische Digitalisierungskritik darstellt, muss hier zunächst offenbleiben.

Gleichwohl sollte deutlich geworden sein, dass die digitale Gegenwart keineswegs dem geschichtslosen Selbstbild entspricht, welches sie von sich selbst gerne zeichnet. Die Entwicklung der Moderne ist auch eine Variationsreihe technikbasierter, vergangener Zukünfte. Um den behaupteten Innovations- und Problemlösungsanspruch nicht durch historischen Quellenreichtum zu verwässern, überschreiben diese Entwürfe ihre historische Genealogie durch personalisierte Gründungserzählungen jedoch beginnt die Geschichte der Digitalisierung nicht erst in Wohnheimzimmern von Ivy-League-Universitäten oder kalifornischen Garagen. Ihre Wurzeln gehen zurück auf die modernen Utopien, die sich von technischer Verhaltenssteuerung eine perfektionierte Ordnung versprochen haben.

Damit kann auch die These ergänzt werden, nach der die digitale Gesellschaft konsequent aus den sozialen Strukturmustern der ausdifferenzierten und binär codierten Moderne hervorgegangen ist (Nassehi 2019): Auch in ihren technokratischen Steuerungsutopien ist die Gegenwart keineswegs durch einen revolutionären Bruch von der bisherigen Moderne getrennt. Diskutiert werden müsste dann, ob beide Genealogien sich ergänzen oder in einem Spannungsverhältnis stehen - genau hier bietet sich die zukünftige Möglichkeit, die Digitalisierungsdebatte zwischen politischer und soziologischer Theorie zu eröffnen. Insofern die behavioristische Revolution der Hochmoderne auch das szientifische Selbstverständnis der Politikwissenschaft massiv geformt hat, müsste sich dann allerdings auch das Fach an dieser Stelle stärker mit seinem technokratischen Erbe und den aus dieser Tradition hervorgegangenen Steuerungsansprüchen beschäftigen - dies kann es aber nur dann leisten, wenn es die in der politischen Theorie verankerte ideengeschichtliche Selbstreflexion nicht abreißen lässt und sich damit die Fähigkeit zur eigenen Kontextualisierung bewahrt.

Funding Open Access funding enabled and organized by Projekt DEAL. 
Open Access Dieser Artikel wird unter der Creative Commons Namensnennung 4.0 International Lizenz veröffentlicht, welche die Nutzung, Vervielfältigung, Bearbeitung, Verbreitung und Wiedergabe in jeglichem Medium und Format erlaubt, sofern Sie den/die ursprünglichen Autor(en) und die Quelle ordnungsgemäß nennen, einen Link zur Creative Commons Lizenz beifügen und angeben, ob Änderungen vorgenommen wurden.

Die in diesem Artikel enthaltenen Bilder und sonstiges Drittmaterial unterliegen ebenfalls der genannten Creative Commons Lizenz, sofern sich aus der Abbildungslegende nichts anderes ergibt. Sofern das betreffende Material nicht unter der genannten Creative Commons Lizenz steht und die betreffende Handlung nicht nach gesetzlichen Vorschriften erlaubt ist, ist für die oben aufgeführten Weiterverwendungen des Materials die Einwilligung des jeweiligen Rechteinhabers einzuholen.

Weitere Details zur Lizenz entnehmen Sie bitte der Lizenzinformation auf http://creativecommons.org/ licenses/by/4.0/deed.de.

\section{Literatur}

Anders, Günther. 1956. Über die Seele im Zeitalter der zweiten industriellen Revolution. Die Antiquiertheit des Menschen, Bd. 1. München: C.H. Beck.

Arendt, Hannah. 1981. Vita activa oder Vom tätigen Leben. München: Piper.

Aristoteles. 1969. Nikomachische Ethik. Stuttgart: Reclam. Übers. v. Franz Dirlmeier.

August, Vincent. 2021. Technologisches Regieren. Der Aufstieg des Netzwerk-Denkens in der Krise der Moderne. Foucault, Luhmann und die Kybernetik. Bielefeld: transcript.

Barbrook, Richard, und Andy Cameron. 1995. The Californian Ideology. https://www.metamute.org/ editorial/articles/californian-ideology. Zugegriffen: 29. Nov. 2021

Barlow, John Perry. 1996. A declaration of the independence of cyberspace. https://www.eff.org/ cyberspace-independence. Zugegriffen: 29. Nov. 2021.

Bates, David William. 2012. States of war. Enlightenment origins of the political. New York: Columbia UP.

Bellamy, Edward. 1980. Ein Rückblick aus dem Jahre 2000. Leipzig: Reclam.

Berg, Sebastian, Sebastian Rakowski, und Thorsten Thiel. 2020. Die digitale Konstellation. Eine Positionsbestimmung. Zeitschrift für Politikwissenschaft 30:171-191.

Blumenberg, Hans. 1983. Die Lesbarkeit der Welt. Frankfurt/M.: Suhrkamp.

Blumenberg, Hans. 1996. Die Legitimität der Neuzeit. Frankfurt/M.: Suhrkamp.

Blumenberg, Hans. 2015. Das Verhältnis von Natur und Technik als philosophisches Problem. In Schriften zur Technik, Hrsg. A. Schmitz, B. Stiegler, 17-29. Berlin: Suhrkamp.

Boltanski, Luc, und Erik Esquerre. 2018. Bereicherung. Eine Kritik der Ware. Berlin: Suhrkamp.

Bredekamp, Horst. 2003. Thomas Hobbes: Der Leviathan. Das Urbild des modernen Staates und seine Gegenbilder. 1651-2001, 2. Aufl., Berlin: Akademie.

Bridle, James. 2019. New Dark Age. Der Sieg der Technologie und das Ende der Zukunft. München: C.H. Beck.

Bröckling, Ulrich. 2017. Gute Hirten führen sanft. Über Menschenregierungskünste. Berlin: Suhrkamp.

Chomsky, Noam. 1959. Verbal Behavior. By B.F. Skinner. Language 35:26-58.

Chomsky, Noam. 1971. The Case against B.F. Skinner. The New York review of Books. https://www. nybooks.com/articles/1971/12/30/the-case-against-bf-skinner/ (Erstellt: 30. Dez. 1971). Zugegriffen: 29. Nov. 2021.

Condorcet. 1993. Arithmétique politique: textes rares ou inédits (1767-1789). Paris: INED. Hrsg. v. Bernard Bru.

Condorcet. 2010. Freiheit, Revolution, Verfassung. Kleine politische Schriften. Berlin: Akademie. Hrsg. v. Daniel Schulz.

Constant, Benjamin. 1972. Über die Freiheit der Alten im Vergleich zu der der Heutigen. Eine Rede vor dem Athénée Royal in Paris. In Politische Schriften Werke in vier Bänden, Bd. 4, Hrsg. Axel Blaeschke, Lothar Gall, 363-396. Berlin: Propyläen.

Cromby, John, und Martin E.H. Willis. 2014. Nudging into subjectification. Governmentality and psychometrics. Critical Social Policy 34:241-259.

Dahrendorf, Ralf. 1965. Pfade aus Utopia. Zu einer Neuorientierung der soziologischen Analyse. In Gesellschaft und Freiheit. Zur soziologischen Analyse der Gegenwart, 85-111. München: Piper. 
Daub, Adrian. 2020. Was das Valley Denken nennt. Über die Ideologie der Tech-Branche. Berlin: Suhrkamp.

Dreischer, Stephan, et al. (Hrsg.). 2013. Jenseits der Geltung. Transzendenzbehauptungen von der Antike bis zur Gegenwart. Berlin, Boston: De Gruyter.

Ellul, Jacques. 1964. The technological society. New York: A. Knopf.

Elster, Jon. 2000. Ulysses unbound. Studies in rationality, precommitment, and constraints. Cambridge: Cambridge UP.

Engels, Friederich. 1973. Die Entwicklung des Sozialismus von der Utopie zur Wissenschaft. In Marx/ Engels Werke, 4. Aufl., Bd. 19, 177-228. Berlin: Dietz.

Erickson, Paul, et al. 2013. How reason almost lost its mind. The strange career of cold war rationality. Chicago:

Etzemüller, Thomas (Hrsg.). 2009. Die Ordnung der Moderne. Social Engineering im 20. Jahrhundert. Bielefeld: transcript.

Etzemüller, Thomas. 2010. Die Romantik der Rationalität. Gunnar \& Alva Myrdal - Social Engineering in Schweden. Bielefeld: transcript.

Falter, Jürgen. 2019. Behaviorismus, Behavioralismus. Staatslexikon. https://www.staatslexikon-online.de/ Lexikon/Behaviorismus,_Behavioralismus. Zugegriffen: 29. Nov. 2021.

Fischer, Karsten. 2014. Überwachen und steuern. Was der Staat nicht wissen darf und auch nicht wissen sollte. Kursbuch 180:45-57.

Floridi, Luciano. 2015. Die 4. Revolution. Wie die Infosphäre unser Leben verändert. Berlin: Suhrkamp.

Fourier, Charles. 1966. Theorie der vier Bewegungen und der allgemeinen Bestimmungen. Frankfurt/M.: Europäische Verlagsanstalt. Hrsg. v. Theodor W. Adorno.

Frankenberg, Günter. 2010. Staatstechnik. Perspektiven auf Rechtsstaat und Ausnahmezustand. Berlin: Suhrkamp.

Frauenholz, Uwe, et al. 2012. Hochmoderne Visionen und Utopien. Zur Transzendenz technischer Fortschrittserwartungen. In Technology Fiction. Technische Visionen und Utopien in der Hochmoderne, Hrsg. Uwe Fraunholz, Anke Woschech, 11-24. Bielefeld: transcript.

Görlitz, Axel, und Hans-Peter Burth. 1998. Politische Steuerung. Ein Studienbuch, 2. Aufl., Opladen: Leske + Budrich.

Guizot, François. 1985. Histoire de la civilization en Europe. Paris: Hachette. Hrsg. v. Pierre Rosanvallon.

Habermas, Jürgen. 1969. Technik und Wissenschaft als ,Ideologie‘. Frankfurt/M.: Suhrkamp.

Heukelom, Floris. 2014. Behavioral economics. A history. Cambridge: Cambridge University Press.

Hirschman, Albert O. 1977. The passions and the interests. Political arguments for capitalism before its triumph. Princeton: Princeton University Press.

Hobbes, Thomas. 1996. Leviathan. Hamburg: Meiner. Hrsg. v. Hermann Klenner.

Huxley, Aldous. 1977. Brave new world. London: Granada.

Kaufmann, Thomas. 2017. Erlöste und Verdammte. Eine Geschichte der Reformation. München: C.H. Beck.

Koestler, Arthur. 1967. The ghost in the machine. London: Hutchinson.

König, Pascal D. 2018. Algorithmen und die Verwaltung sozialer Komplexität. Zur Neukonfigurierung der Idee der Selbstregulierung des Volkes. Zeitschrift für Politikwissenschaft 28:289-312.

Koselleck, Reinhart. 1979. Vergangene Zukunft. Zur Semantik geschichtlicher Zeiten. Frankfurt/M.: Suhrkamp.

Koselleck, Reinhart. 1985. Die Verzeitlichung der Utopie. In Utopieforschung, Bd. 3, Hrsg. v. Wilhelm Voßkamp, 1-15. Frankfurt/M.: Suhrkamp.

Koster, Ann-Kathrin. 2021. Das Ende des Politischen? Demokratische Politik und Künstliche Intelligenz. Zeitschrift für Politikwissenschaft https://doi.org/10.1007/s41358-021-00280-5.

Krause, Marcus. 2007. The Question of Control. Die Erziehung der Gesellschaft bei Huxley, Orwell und Skinner. In Jenseits von Utopie und Entlarvung. Kulturwissenschaftliche Untersuchungen zum Erziehungsdiskurs in der Moderne, Hrsg. Eva Geulen, Nicolas Pethes, 179-204. Freiburg: Rombach.

Kruft, Hanno-Walter. 1989. Städte in Utopia. Die Idealstadt vom 15. bis zum 18. Jahrhundert. München: C.H. Beck.

Kuhlmann, Hilke. 2005. Living Walden two. B.F. Skinner's behaviorist utopia and experimental communities. Urbana: University of Illinois Press.

Kurzweil, Ray. 2005. Singularity is near. When humans transcend biology. New York: Viking.

Lange, Stefan. 2002. Die politische Utopie der Gesellschaftssteuerung. In Theorie der Politik. Niklas Luhmanns politische Soziologie, Hrsg. Kai-Uwe Hellmann, Rainer Schmalz-Bruns, 171-193. Berlin: Suhrkamp.

Laswell, Harold D. 1936. Politics. Who gets what, when, and how? New York, London: McGraw-Hill. 
Lepenies, Robert, und Magdalena Malecka. 2015. The institutional consequences of nudging - nudges, politics, and law. Review of Philosophy and Psychology 6:427-437.

Lepore, Jill. 2020. If then. How the Simulmatics corporation invented the future. New York: Liveright.

Loewenstein, Bedrich. 2015. Der Fortschrittsglaube. Europäisches Geschichtsdenken zwischen Utopie und Ideologie. Darmstadt: Wissenschaftliche Buchgesellschaft.

Machiavelli, Niccolò. 1990. Der Fürst. Herausgegeben von Horst Günther. Frankfurt/M.: Insel.

Malecka, Magdalena. 2021. Knowledge, behaviour, and policy: questioning the epistemic presuppositions of applying behavioural science in public policymaking. Synthese https://doi.org/10.1007/s11229021-03026-6.

Manuel, Frank E. und Fritzie P. Manuel, 1979. Utopian Thought in the Western World. Cambridge: Harvard UP.

Meynaud, Jean. 1964. La téchnocratie. Mythe où réalité? Paris: Payot.

Morozow, Evgeny. 2013. Smarte Neue Welt. Digitale Technik und die Freiheit des Menschen. München: Blessing.

Mühlhoff, Rainer, et al. (Hrsg.). 2019. Affekt, Macht, Netz. Auf dem Weg zu einer Sozialtheorie der Digitalen Gesellschaft. Bielefeld: transcript.

Mumford, Lewis. 1977. Mythos der Maschine. Kultur, Technik und Macht. Frankfurt/M.: Fischer.

Nassehi, Armin. 2002. Politik des Staates oder Politik der Gesellschaft? Kollektivität als Problemformel des Politischen. In Theorie der Politik. Niklas Luhmanns politische Soziologie, Hrsg. v. Kai-Uwe Hellmann, Rainer Schmalz-Bruns, 38-59. Berlin: Suhrkamp.

Nassehi, Armin. 2019. Muster. Theorie der digitalen Gesellschaft. München: C.H. Beck.

Naudé, Gabriel. 2004. Considérations politiques sur les coups d'État. Paris: Gallimard. Hrsg. v. Frédérique Marin und Marie-Odile Perulli.

Negroponte, Nicholas. 1995. Being digital. New York: Vintage.

Nosthoff, Anna-Verena, und Felix Maschewski. 2019. The obsolescence of politics: rereading Günther Anders's critique of cybernetic governance and integral power in the digital age. Thesis Eleven 153:75-93.

Ottmann, Henning. 2006. Geschichte des politischen Denkens. Bd. 3/1. Stuttgart: Metzler.

Pentland, Alex. 2015. Social physics. How social networks can make us smarter. London: Penguin.

Pohle, Jörg, et al. 2018. Nudging, Regulierung durch Big Data und Verhaltenswissenschaften. Berlin: Abida.

Quetelet, Adolphe. 1835. Sur l'homme et le developpement de ses facultés, ou essai de physique sociale. Paris: Bachelier. 2 Bde.

Ramm, Thilo (Hrsg.). 1968. Der Frühsozialismus, 2. Aufl., Stuttgart: Kröner.

Reckwitz, Andreas. 2017. Die Gesellschaft der Singularitäten. Berlin: Suhrkamp.

Rheingold, Howard. 1993. The virtual community. Homesteading on the electronic frontier. Reading: Addison Wesley.

Rohe, Karl. 1994. Politik. Begriffe und Wirklichkeiten, 2. Aufl., Stuttgart: Kohlhammer.

Rosa, Hartmut. 2018. Unverfügbarkeit. Wien, Salzburg: Residenz.

Rosanvallon, Pierre. 1979. Le capitalism utopique. Critique de l'idéologie économique. Paris: Seuil.

Rosanvallon, Pierre. 2015. Le bon gouvernement. Paris: Seuil.

Rousseau, Jean-Jacques. 1995. Über den Ursprung der Ungleichheit unter den Menschen. In Rousseau, Jean-Jacques. Schriften zur Kulturkritik, 5. Aufl., Hrsg. Kurt Weigang, 77-268. Hamburg: Meiner.

Saage, Richard. 1997. Utopieforschung. Eine Bilanz. Darmstadt: Primus.

Saage, Richard. 2002. Utopische Profile. Industrielle Revolution und Technischer Staat im 19. Jahrhundert. Münster: LIT.

Schelsky, Helmut. 1979. Der Mensch in der technischen Zivilisation. In Auf der Suche nach Wirklichkeit. Gesammelte Aufsätze zur Soziologie der Bundesrepublik, 449-499. München: Goldmann.

Schulz, Daniel. 2015. Die Krise des Republikanismus. Baden-Baden: Nomos.

Schulz, Daniel. 2017. Verfassungsbilder: Zur Differenz von Körper und Text. In Leviathan 45, Sonderband 34: Politische Ikonographie und Differenzrepräsentation, Hrsg. Sebastian Huhnholz, Eva Hausteiner, 71-93.

Scott, James C. 1999. Seeing like a state. How certain schemes to improve the human condition have failed. New Haven: Yale UP.

Skinner, Burrhus F. 1953. Science and human behavior. New York: Macmillan.

Skinner, Burrhus F. 1971a. Futurum zwei. Die Vision einer aggressionsfreien Gesellschaft. Reinbek: Rowohlt.

Skinner, Burrhus F. 1971b. Beyond freedom and dignity. Indianapolis, Cambridge: Hackett.

Skinner, Burrhus F. 1974. About behaviorism. New York: Knopf.

Skinner, Burrhus F. 1976. Walden two. Indianapolis, Cambridge: Hackett. 
Skinner, Quentin. 1996. Reason and rhetoric in the philosophy of hobbes. Cambridge: Cambridge University Press.

Skinner, Quentin. 1998. The foundation of modern political thought. Bd. 1. Cambridge: Cambridge University Press.

Skinner, Quentin. 2017. Hobbes und die Person des Staates. Berlin: Duncker\&Humblot.

Stalder, Felix. 2016. Kultur der Digitalität. Berlin: Suhrkamp.

Stollberg-Rilinger, Barbara. 1986. Der Staat als Maschine. Zur politischen Metaphorik des absoluten Fürstenstaats. Berlin: Duncker\&Humblot.

Strohschneider, Peter. 2014. Zur Politik der Transformativen Wissenschaft. In Die Verfassung des Politischen, Hrsg. André Brodocz, et al., 175-192. Wiesbaden: Springer.

Sunstein, Cass. 2015. Choosing not to choose. Understanding the value of choice. Oxford: Oxford University Press.

Swirski, Peter. 2011. American utopia and social engineering in literature, social thought, and political history. New York: Routledge.

Taylor, Charles. 1964. The explanation of behaviour. London: Routledge \& Keagan Paul.

Thaler, Richard H., und Cass R. Sunstein. 2003. Libertarian paternalism. The American Economic Review 93:175-179.

Thaler, Richard H., und Cass R. Sunstein. 2009. Nudge. Wie man kluge Entscheidungen anstößt. Berlin: Econ.

Thiel, Thorsten. 2014. Die Schönheit der Chance: Utopien und das Internet. Juridikum: Zeitschrift für Kritik, Recht, Gesellschaft 15:459-471.

Turkle, Sherry. 1984. Die Wunschmaschine. Vom Entstehen der Computerkultur. Reinbek: .

Turner, Fred. 2006. From counterculture to cyberculture. Stewart brand, the whole earth network, and the rise of digital utopianism. Chicago: Chicago UP.

Ulbricht, Lena. 2020. Scraping the demos. Digitalization, web scraping and the democratic project. Democratization 27:426-442.

Veblen, Thorstein. 1921. The engineers and the price-system. New York: Huebsch.

Vorländer, Hans (Hrsg.). 2013. Transzendenz und die Konstitution von Ordnung. Berlin, Boston: De Gruyter.

Voßkamp, Wilhelm (Hrsg.). 1985. Utopieforschung. Interdisziplinäre Studien zur neuzeitlichen Utopie. Frankfurt/M.: Suhrkamp. 3 Bde.

Watson, John B. 1913. Psychology as the behaviorist views it. Psychological Review 20:158-177.

Weber, Max. 2009. Wirtschaft und Gesellschaft. Herrschaft. Max Weber-Gesamtausgabe, Bd. 1, 22-24. Tübingen: Mohr Siebeck.

Weiser, Mark. 1991. The computer for the 21st century. https://www.ics.uci.edu/ corps/phaseii/WeiserComputer21stCentury-SciAm.pdf. Zugegriffen: 29. Nov. 2021.

Weizenbaum, Joseph. 1977. Die Macht der Computer und die Ohnmacht der Vernunft. Frankfurt/M.: Suhrkamp.

Willeke, Stefan. 1995. Die Technokratiebewegung in Nordamerika und Deutschland zwischen den Weltkriegen. Eine vergleichende Analyse. Frankfurt/M.: Peter Lang.

Wolpert, Rita S. 2005. A multicultural feminist analysis of Walden two. The Behaviour Analyst Today 6:186-190.

Zuboff, Shoshana. 2018. Das Zeitalter des Überwachungskapitalismus. Frankfurt, New York: Campus. 